Journal of Environmental
AnSN: $2525-815 \mathrm{X}$

\title{
Estrutura morfológica foliar da arborização urbana na manutenção do conforto térmico
}

\section{Morphological leaf structure of urban arborization in the maintenance of thermal comfort}

\author{
Luiz Henrique Gonçalves da Silva ${ }^{\mathrm{a}}$, Rejane Magalhães de Mendonça Pimentel ${ }^{\mathrm{a}}$ \\ ${ }^{\text {a }}$ Universidade Federal Rural de Pernambuco-UFRPE, Laboratório de Fitomorfologia Funcional-LAFF, Rua Dom Manoel \\ de Medeiros, SN, Dois Irmãos, Pernambuco, Brasil. CEP: 52171-900. E-mail: luizzhenrique.g@gmail.com, \\ rejanemmpimentel@gmail.com.
}

\begin{tabular}{l}
\hline A R T I C L E I N F O \\
\hline Recebido 03 Jan 2019 \\
Aceito 28 Jan 2019 \\
Publicado 31 Jan 2019
\end{tabular}

\begin{abstract}
A B S T R A C T
The increase in population density in urban centers intensifies the effects of urbanization, causing an increase in temperature, providing thermal discomfort in the local population. The squares and urban parks have proved to be a viable alternative, having a good cost/benefit ratio. In the squares, the trees are the main components for the mitigation of the temperature and increase of the relative humidity of the air through its foliage. The different tissues that compose the leaf react differently as the absorption dynamics of solar radiation, implying a greater or lesser amount of absorbed radiation. The objective of this study is to evaluate the contribution of vegetation in squares for the maintenance of thermal comfort and how this contribution is related to leaf morphology. The temperature and relative humidity of the air below the canopy and outside the canopy formation limit of five species established in an urban center square was measured. Leaves were collected for morphological analysis and data were obtained on the temperature and relative humidity of the air, immediately below the crown and outside the projection limit of the same on the soil surface. All the species investigated showed a greater thermal comfort below the canopy. However, the ones that had the highest temperature sensitivity showed a greater specific leaf area, being more efficient in the absorption of solar radiation, informing about the importance of the urban afforestation in maintaining the quality of life in cities.
\end{abstract}

Keywords: Climate regulation, environmental services, urban comfort.

\section{R E S U M O}

O aumento na densidade demográfica nos centros urbanos intensifica os efeitos da urbanização, ocasionando o aumento na temperatura, proporcionando desconforto térmico na população local. As praças e parques urbanos mostraram-se uma alternativa viável, possuindo um bom custo/benefício. Nas praças, as árvores são os principais componentes para a amenização da temperatura e aumento da umidade relativa do ar através de sua folhagem. Os diferentes tecidos que compõem a folha reagem de maneira diferente quanto a dinâmica de absorção da radiação solar, implicando em uma maior ou menor quantidade de radiação absorvida. O objetivo desse estudo é avaliar a contribuição da vegetação em praças para a manutenção do conforto térmico e como essa contribuição está relacionada à morfologia foliar. Foi medida a temperatura e umidade relativa do ar abaixo da copa e fora do limite de formação da copa de cinco espécies estabelecidas em praça de centro urbano. Foram coletadas folhas para análise morfológica e obtidos dados da temperatura e umidade relativa do ar, imediatamente abaixo da copa e fora do limite de projeção da mesma na superfície do solo. Todas as espécies investigadas mostraram contribuição para um maior conforto térmico abaixo de sua copa, entretanto as que mais amenizaram a temperatura apresentaram maior área foliar específica, sendo mais eficaz na absorção 
de radiação solar, informando sobre a importância da arborização urbana na manutenção da qualidade de vida nas cidades.

Palavras-Chave: Regulação do clima, serviços ambientais, conforto urbano.

\section{Introdução}

A crescente urbanização tem conduzido a uma drástica redução da vegetação nativa. Isto tem modificado a estrutura de cobertura do solo e vem causando desconfortos para a população humana que utiliza aquele ambiente.

Dentre os principais desconfortos provenientes dessa crescente urbanização destaca-se o aumento na temperatura ambiente, provocando o fenômeno denominado ilhas de calor, causando um desequilíbrio térmico prejudicial ao bem-estar humano (Huang et al., 2019).

Diversas formas de mitigar os efeitos da urbanização vem sendo desenvolvidas, dentre elas estão os parques e praças em ambiente urbano (Aboelata, 2017; Solecki et al., 2011). A vegetação presente nessas áreas é capaz de amenizar o desconforto térmico devido a sua capacidade de absorver radiação solar e liberar água para a atmosfera circundante por evapotranspiração, contribuindo com a prestação de serviços ambientais, através da amenização na temperatura e o aumento na umidade relativa do ar, respectivamente (Aboelata, 2017; Damalgo et al., 2006).

Considerando a vegetação estabelecida em parques e praças, as árvores são capazes de proteger o solo com a projeção de sua copa, contribuindo para a formação de um microclima agradável. Mesmo possuindo diversos agentes dificultadores, como escassez de nutrientes e água no solo (devido ao fato dessas plantas receberem água exclusivamente da precipitação), muitas espécies de porte arbóreo se mostram aptas a um perfeito estabelecimento nesses locais.

A copa das árvores desempenha papel fundamental na amenização de fatores climatológicos associados ao conforto térmico em centros urbanos. As árvores produzem folhas com menor área após longos períodos com altas temperaturas e precipitação reduzida ou ausente por períodos prolongados (Kardel et al., 2010; Zhao \& Running, 2010). Esta condição ambiental leva a planta a uma condição de estresse hídrico e aumento nas taxas de evapotranspiração. A altura do início de formação da copa, em relação à superfície do solo, é um caractere que irá influenciar na velocidade do vento e, desta forma, modifica o movimento do vapor de água e altera a umidade relativa do ar nas imediações destas plantas.

As folhas apresentam grande plasticidade quanto à sua forma e capacidade de uso da água na assimilação/alocação de fotoassimilados (Araujo et al., 2017). O conhecimento das variações relativas a estes aspectos permite avaliar o perfil funcional das espécies, especialmente daquelas estabelecidas sob determinadas condições ambientais, informando sobre a contribuição na amenização de desconfortos causados pela urbanização. Ao mesmo tempo, contribui para uma avaliação do comportamento espectral relativo à absorbância da irradiação solar.

O estudo objetiva identificar a influência das características morfológicas foliares, de espécies arbóreas estabelecidas em área urbana, na capacidade de amenização do desconforto térmico provocado por elevados valores de temperatura e umidade relativa do ar.

\section{Material e Métodos}

Área de estudo e seleção de espécies

$\mathrm{O}$ estudo foi realizado uma área urbanizada na cidade do Recife-PE (Figura 1).

A vegetação arbórea investigada se constituiu de cinco árvores, de espécies distintas, com Diâmetro na Altura do Peito (DAP) maior que $10 \mathrm{~cm}$, estabelecidas na Praça Farias Neves, em Dois Irmãos, Recife-PE, com auxílio de trena milimetrada.

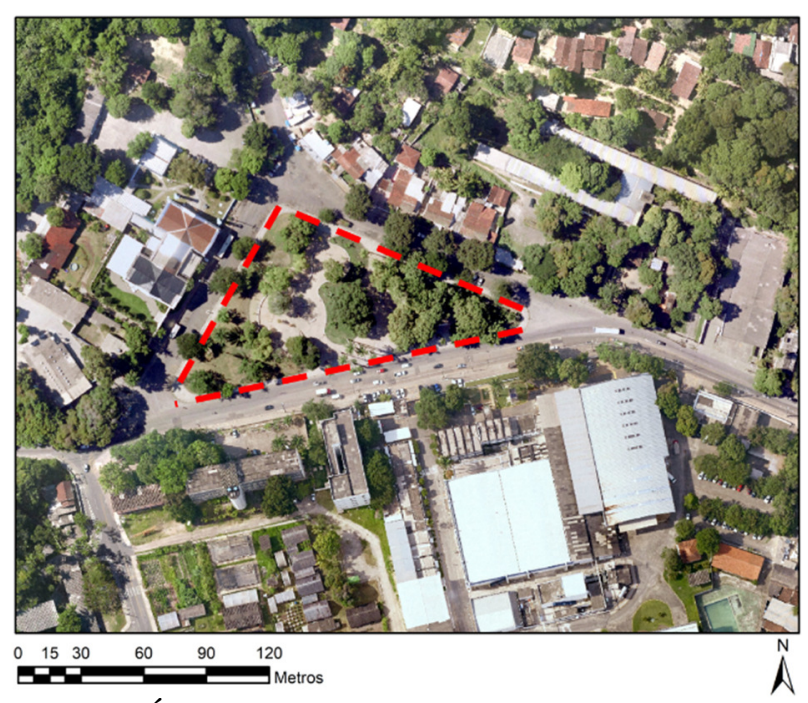

Figura 1. Área de estudo (tracejada) na Praça Farias Neves, Dois Irmãos, Recife-PE. Fonte: Adaptado do QuickBird (2013).

\section{Identificação taxonômica das espécies arbóreas}

A identificação taxonômica das espécies foi realizada com auxílio de bibliografia especializada, comparação com material de herbário e consulta a especialistas, seguindo o sistema de classificação APG IV (2016).

Determinação da temperatura do ar, umidade relativa do ar e distância entre início da copa e solo 
A obtenção de informações sobre a influência das árvores na amenização da temperatura e umidade relativa do ar considerou pontos imediatamente abaixo da projeção da copa das árvores $\left(\mathrm{T}_{\mathrm{a}}\right.$ e $\left.\mathrm{UR}_{\mathrm{a}}\right) \mathrm{e}$ áreas próximas $\left(T_{p}\right.$ e $\left.U_{p}\right)$, sob plena iluminação natural, sem a influência das copas (Figura 2). Estes dados foram obtidos com a utilização de um termohigrômetro (Incoterm ${ }^{\circledR}$ ).

A contribuição do sombreamento da copa das árvores na amenização das condições ambientais foi determinada através da subtração dos valores obtidos para a temperatura $\left(\mathrm{Tp}_{\mathrm{a}}-\mathrm{T}_{\mathrm{a}}\right)$ e umidade relativa do ar $\left(\mathrm{UR}_{\mathrm{p}}-\mathrm{UR}_{\mathrm{a}}\right)$, denominado como Diferença de Temperatura (DT).

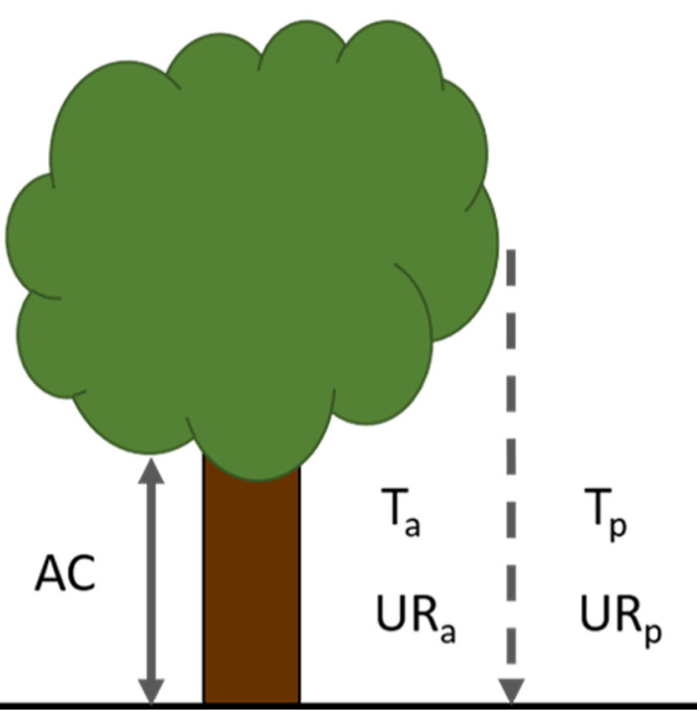

Figura 2. Esquema mostrando pontos de amostragem da coleta de dados abióticos da Temperatura (T) e Umidade Relativa do ar (UR), imediatamente abaixo $\left(T_{a}\right.$ e $U_{a}$ ), além da projeção da copa na superfície do solo $\left(T_{p}\right.$ e $\left.U_{\mathrm{p}}\right)$ e altura do limite inferior da copa em relação à superfície do solo.

\section{Amostragem e processamento de folhas}

De cada espécie foram coletadas 10 folhas maduras, expostas ao sol; foram consideras folhas maduras aquelas localizadas entre o quinto e sétimo nó de um ramo. As amostras foram levadas ao
Laboratório de Fitomorfologia Funcional (LAFF), da Universidade Federal de Pernambuco (UFRPE), para realização das análises.

Determinação da área foliar e área foliar específica

Metade das amostras foram digitalizadas em scanner de mesa, para posterior análise dos caracteres morfológicos: Área Foliar (AF) $\left(\mathrm{cm}^{2}\right)$ e Área Foliar Específica (AFE) $\left(\mathrm{cm}^{2} \cdot \mathrm{g}^{-1}\right)$.

Determinação de massa fresca e seca e teor relativo de água

A determinação dos valores de massa fresca foi realizada através da pesagem, em balança digital (duas casas decimais).

Os valores de massa seca foram obtidos após secagem das folhas em estufa, por $48 \mathrm{~h}$, a $60^{\circ} \mathrm{C}$ e pesagem em balança digital (duas casas decimais).

O Teor Relativo de Água (TRA) foi obtido através da subtração do peso de massa fresca (PMF) pelo peso de massa seca (PMS).

\section{Análise estatística}

A análise estatística dos dados foi realizada usando o software R (R CORE TEAM, 2018). A variação nos dados relativos aos caracteres morfológicos foliares foi determinada com a aplicação do teste ANOVA.

\section{Resultados}

Considerando o diâmetro do tronco na altura do peito, foram selecionadas espécies arbóreas representantes de diferentes famílias botânicas (Tabela 1). Todas as espécies apresentaram folhas simples, exceto $H$. impetiginosus, que possui folhas compostas, digitadas, com cinco folíolos.

O maior e menor valores para o DAP foram determinados em Licania tomentosa e Handroanthus impetiginosus, respectivamente (Tabela 1).

Os valores obtidos para a altura do limite inferior da copa em relação à superfície do solo foram semelhantes para quatro espécies, diferindo apenas para Artocarpus heterophyllus (Tabela 1).

Tabela 1. Lista de espécies de árvores, seguida da família, nome vulgar e Diâmetro na Altura do Peito (DAP) e altura do limite inferior da copa em relação à superfície do solo (AC), estabelecidas na Praça Farias Neves, em Dois Irmãos, Recife-PE.

\begin{tabular}{lllcc}
\hline Espécie & Família & Nome vulgar & DAP (cm) & AC (m) \\
\hline Syzygium malaccense (L.) Alston & Myrtaceae & Jambeiro & 29,3 & 2,58 \\
Mangifera indica L. & Anacardiaceae & Mangueira & 44,59 & 2,81 \\
Licania tomentosa (Benth.) Fritsch. & Chrysobalanaceae & Oitizeiro & 56,53 & 2,89 \\
Handroanthus impetiginosus (Vell.) Mattos & Bignoniaceae & Ipê-roxo & 17,20 & 2,37 \\
Artocarpus heterophyllus L. & Moraceae & Jaqueira & 33,44 & 5,00 \\
\hline
\end{tabular}

Os menores e maiores valores de $\mathrm{T}_{\mathrm{a}}$ e $\mathrm{UR}_{\mathrm{p}}$, respectivamente, foram encontrados abaixo da copa de H. impetiginosus (Tabela 2). Os valores obtidos para $\mathrm{T}_{\mathrm{p}}$ diferiram próximo a $1^{\circ} \mathrm{C}$ para duas das espécies em estudo (Tabela 2). 
Considerando as diferenças na temperatura e umidade relativa do ar, abaixo da copa e área fora do sombreamento da copa sobre a superfície do solo, os maiores valores obtidos para DT e DUR foram mensurados em $S$. malaccence e $H$. impetiginosus (Tabela 2).

Tabela 2. Temperatura e umidade relativa do ar na Praça Farias Neves, Dois irmãos, Recife-PE. $T_{p}=$ Temperatura em área exposta à radiação e próxima à extremidade da copa; $\mathrm{T}_{\mathrm{a}}=$ Temperatura abaixo da copa; $\mathrm{DT}=$ Diferença de Temperatura; $\mathrm{UR}_{\mathrm{p}}=$ Umidade Relativa do ar em área exposta à radiação e próxima à extremidade da copa; $\mathrm{UR}_{\mathrm{a}}=$ Umidade Relativa abaixo da copa; DUR = Diferença de Umidade Relativa.

\begin{tabular}{|c|c|c|c|c|c|c|}
\hline \multirow{2}{*}{ Espécie } & $\mathbf{T p}$ & Ta & DT & URp & URa & DUR \\
\hline & \multicolumn{3}{|c|}{$\mathbf{C}$} & \multicolumn{3}{|c|}{$\%$} \\
\hline Handroanthus impetiginosus & 33,0 & 31,0 & 2,0 & 40 & 43 & -3 \\
\hline Mangifera indica & 34,4 & 32,4 & 2,0 & 40 & 41 & -1 \\
\hline Syzygium malaccense & 33,8 & 31,1 & 2,7 & 42 & 42 & - \\
\hline Licania tomentosa & 34,0 & 31,8 & 2,2 & 40 & 42 & 2 \\
\hline Artocarpus heterophyllus & 34,5 & 32,7 & 1,8 & 37 & 37 & - \\
\hline
\end{tabular}

Handroanthus impetiginosus apresentou os maiores valores para AF, PMF, PMS e TRA, acompanhados de maiores variações no desvio padrão das médias (Tabela 3).
Licania tomentosa e $S$. malaccence apresentaram maiores valores para a AFE (Tabela 3). A primeira espécie se destacou por apresentar os menores valores para AF, PMF, PMS e TRA (Tabela $3)$.

Tabela 3. Parâmetros morfológicos foliares de espécies arbóreas utilizadas na arborização urbana do Recife. $\mathrm{AF}=$ Área Foliar; AFE = Área Foliar Específica; PMF = Peso de Massa Fresca; PMS = Peso de Massa Seca; TRA $=$ Teor Relativo de Água.

\begin{tabular}{|c|c|c|c|c|c|}
\hline \multirow{2}{*}{ Espécie } & $\mathbf{A F}$ & AFE & PMF & PMS & TRA \\
\hline & $\mathrm{cm}^{2}$ & $\mathrm{~cm}^{2} \cdot \mathrm{g}-1$ & $\mathrm{G}$ & $\mathrm{g}$ & $\%$ \\
\hline Handrc & $477,94 \pm 30,60 \mathrm{a}$ & $106,98 \pm 8,05 a$ & $9,74 \pm 1,19^{a}$ & $4,44 \pm 0,42 \mathrm{a}$ & $52,67 \mathrm{~b}$ \\
\hline Mangifera indica & $126,16 \pm 18,04 b$ & $61,20 \pm 2,68 \mathrm{a}$ & $4,21 \pm 0,82 b$ & $1,99 \pm 0,39 a$ & $51,57 \mathrm{~b}$ \\
\hline Syzygium malaccense & $106,93 \pm 7,11 b$ & $117,55 \pm 16,43 a$ & $3,55 \pm 0,44 b$ & $0,93 \pm 0,12 \mathrm{a}$ & $73,89 \mathrm{a}$ \\
\hline Licania tomentosa & $21,80 \pm 5,47 \mathrm{c}$ & $131,15 \pm 35,7 \mathrm{a}$ & $0,37 \pm 0,11 \mathrm{~d}$ & $0,18 \pm 0,06 a$ & $56,64 \mathrm{~b}$ \\
\hline Artocarpus heterophyllus & $54,27 \pm 8,14 \mathrm{c}$ & $93,58 \pm 47,19 a$ & $2,02 \pm 0,29 \mathrm{c}$ & $0,51 \pm 0,26 a$ & $64,97 \mathrm{ab}$ \\
\hline
\end{tabular}

Os dados são medias \pm desvio padrão de cinco repetições por medida, por espécie. Valores na mesma coluna seguido pela mesma letra não foram significativamente diferentes a $\mathrm{P}<0,05$ de acordo com ANOVA.

Considerando as maiores variações no desvio padrão das médias para $\mathrm{AFE}$ e $\mathrm{AF}$, destacaram-se as espécies A. heterophyllus e $H$. impetiginosus (Tabela 3).

Analisando a variação nas médias de AF e PMF, $H$. impetiginosus se destacou com o maior valor significativo, seguido de $M$. indica e $S$. malaccense (Tabela 3).

Não foram encontradas diferenças significativas quanto à AFE e PMS para as espécies analisadas (Tabela 3).

A percentagem do TRA determinado nas folhas foi significativa para $S$. malaccense e A. heterophyllus, seguidas das demais espécies (Tabela 3).

\section{Discussão}

A amenização do conforto térmico atmosférico é proporcionada pela presença de árvores em áreas urbanas (Chun \& Guldmann, 2018). Isto é particularmente dependente da quantidade de árvores e características das espécies que contribuem para a configuração da estrutura das copas, quanto à densidade das folhas (Hu et al., 2018) e consequente grau de sombreamento da superfície do solo.

Áreas urbanizadas com maior presença de árvores promovem melhor qualidade de saúde para a população humana, especialmente quando consideramos as condições do microclima circundante (Hu et al., 2018).

A crescente construção de edificações, associada a uma supressão da vegetação, particularmente de árvores, tem proporcionado a existência das "ilhas de calor" (Ballinas \& Barradas, 2016).

Uma vez que os caracteres foliares informam sobre a condição e resposta das plantas aos fatores do ambiente, o conhecimento das variações relativas à sua plasticidade contribui para ações de manejo e seleção de espécies capazes de minimizar as condições adversas de temperatura e umidade relativa do ar (Marando et al., 2019). 
A maior amenização da temperatura encontrada na área imediatamente abaixo da copa de $H$. impetiginosus deve-se, provavelmente, ao fato desta espécie possuir maior AF, resultando em uma maior área de cobertura do solo. Entretanto, isto não significou um maior TRA para esta espécie. Uma maior expansão da lâmina foliar não implica em um maior TRA, outros caracteres foliares, como a espessura deste órgão, estão mais relacionados à capacidade de armazenamento de água.

Árvores com uma maior densidade de folhas, associada com a arquitetura da copa, contribuem mais para melhores condições climatológicas e, consequente maior conforto térmico.

Apesar do fato de todas as espécies apresentarem amenização na temperatura abaixo de sua copa, as duas que apresentaram maior DT não são indicadas para a arborização de parques e praças, devido à altura de seus indivíduos, pois podem atingir a rede de energia elétrica, causando problemas e acidentes (SMAS, 2013), além da dificuldade para realização dos tratos culturais.

Os parâmetros avaliados nesse estudo estão estritamente relacionados ao funcionamento fisiológico destas plantas, e o resultado pode ser inferido através da AFE. Este caractere evidencia a capacidade das folhas relativa a uma maior alocação de biomassa por unidade de área.

A capacidade de produção/alocação de biomassa nas folhas é medida por meio da AFE, a qual depende de características da estruturação anatômica foliar. A variação no número de camadas e respectiva área transversal ocupada por tecidos fotoassimiladores influenciam fortemente na capacidade de produção/alocação de biomassa na folha (Costa et al., 2015).

As plantas estabelecidas em centros urbanos geralmente investem menos energia no desenvolvimento da AF, pois suas folhas amadurecem mais rápido, em resposta da diminuição de sua longevidade, devido às adversas condições ambientais (Balasooriya et al., 2009). Áreas urbanas se caracterizam por apresentar elevada temperatura do ar, o que acelera o metabolismo celular e consequente amadurecimento precoce das folhas.

\section{Conclusão}

A presença da vegetação auxilia na manutenção do conforto térmico de centros urbanos, diminuindo a temperatura e mantendo reduzida umidade relativa do ar nas proximidades abaixo de suas copas.

Menores valores de AF informam sobre os efeitos negativos da urbanização na reduzida expansão da lâmina foliar, em consequência de elevadas temperaturas. Ao mesmo tempo, maiores valores de AFE informam sobre a capacidade funcional e a prestação de serviços ambientais de árvores estabelecidas em centros urbanos, sob a influência da alteração de fatores climatológicos.

Árvores que desenvolvem folhas com maior área foliar contribui mais para menores valores de temperatura abaixo e nas proximidades da copa.

\section{Agradecimentos}

Agradecemos ao $\mathrm{CNPq}$ pela bolsa de produtividade e de iniciação científica de membros da autoria deste artigo e a equipe de pessoas que compõem o LAFF-UFRPE.

\section{Referências}

ABOELATA, A. A. A. 2017. Study the Vegetation as Urban Strategy to Mitigate Urban Heat Island in Mega City Cairo. Procedia Environmental Sciences, v. 37, p. 386-395.

APG IV. 2016. An update of the Angiosperm Phylogeny Group classification for the orders and families of flowering plants: APG IV. Botanical Journal of the Linnean Society, v. 181, p. 1-2.

ARAUJO, V. K. R.; SANTOS, J. M. F. F.; ARAÚJO, E. L.; PIMENTEL, R. M. M.; SILVA, K. A. 2017. Influence of leaf morphometric variations on the growth of seedlings and juveniles of woody species in a semiarid environment. Brazilian Journal of Botany, v. 40, n. 4, p. 10191028.

BALASOORIYA, B. L. W. K.; SANSOM, R.; MBIKWA, F.; VITHARANA, U.W.A.; BOECKX, P.; VAN MEIRVENNE, M. 2009. Biomonitoring of urban habitat quality by anatomical and chemical leaf characteristics. Environmental and Experimental Botany, v. 65, n. 2-3, p. 386-394.

BALLINAS, M.; BARRADAS, V. L. 2016.The Urban Tree as a Tool to Mitigate the Urban Heat Island in Mexico City: A Simple Phenomenological Model. Journal of Environmental Quality, v. 45, p. 157-166.

CHUN, B.; GULDMANN, J. M. 2018. Impact of greening on the urban heat island: Seasonal variations and mitigation strategies. Computers, Environment and Urban Systems, v. 71, p. 165176. 
COSTA, V. B. S.; SILVA, W. J. M. S.; ALMEIDA, G. M. A.; Ferreira, M. H. G.; OLIVEIRA, T. H.; GALVÍNCIO, J. D.; PIMENTEL, R. M. M. 2015. Influence of Air Pollution in Terminalia catappa L. in Urban Areas. Revista Brasileira de Geografia Física, v. 8, n. 2, p. 236-252.

HU, R.; BOURNEZ, E.; CHENG, S.; JIANG, H.; NERRY, F.; LANDES, T.; SAUDREAU, M.; KASTENDEUCH, P.; NAJJAR, G.; COLIN, J.; YAN, G. 2018. Estimating the leaf area of an individual tree in urban areas using terrestrial laser scanner and path length distribution model. ISPRS Journal of Photogrammetry Remote Sensing, v. 144, p. $357-368$.

HUANG, Q.; HUANG, J.; YANG, X.; FANG, C.; LIANG, Y. 2019. Quantifying the seasonal contribution of coupling urban land use types on Urban Heat Island using Land Contribution Index: A case study in Wuhan, China. Sustainable Cities and Society, v. 44, p. 666-675.

KARDEL, F.; WUYTS, K.; BABANEZHAD, M.; VITHARANA, U.W.A.; WUYTACK, T.; POTTERS, G.; SAMSON, R. 2010. Assessing urban habitat quality based on specific leaf area and stomatal characteristics of Plantago lanceolata L. Environmental Pollution, v. 158, p. 788-794.
MARANDO, F.; SALVATORI, E.; SEBASTIANI, A.; FUSARO, L.; MANES, F. 2019. Regulating Ecosystem Services and Green Infrastructure: assessment of Urban Heat Island effect mitigation in the municipality of Rome, Italy. Ecological Modeling, v. 392, p. 92-102.

R CORE TEAM. 2018. Development Core Team. $\mathrm{R}$ : A language and environment for statistical computing. $\mathrm{R}$ Foundation for Statistical Computing, Vienna, Austria. Disponível em: http://www.R-project.org/.

SMAS. 2013. Secretaria de Meio Ambiente e Sustentabilidade Manual de arborização: orientações e procedimentos técnicos básicos para a implantação e manutenção da arborização da cidade do Recife 1. Ed. Recife. 71p.

SOLECKI, W. D.; ROSENZWEIG, C.; PARSHALL, L.; POPE, G.; CLARK, M.; COX, J. WIENCKE, M. 2005. Mitigation of the heat island effect in urban New Jersey. Environmental Hazards, v. 6, n. 1, p. 39-49.

ZHAO, M.; RUNNING, S. W. 2010. DroughtInduced Reduction in Global Terrestrial Net Primary Production from 2000 Through 2009. Science, v. 329, n. 5994, p. 940-943. 